\title{
Procrastinación académica y la formación de la identidad en los adolescentes de una institución educativa.
}

\section{Academic procrastination and identity formation in adolescents from an educational institution.}

\section{Procrastinación académica}

\author{
Yessenia Vasconez-Mejía ${ }^{1}$ \\ Máximo Tubay-Moreira² \\ Vanessa Betancourt-Zambrano ${ }^{3}$ \\ Mario Cedeño-Yepez ${ }^{4}$ \\ ${ }^{1}$ Universidad Técnica de Babahoyo Extensión Quevedo. Quevedo-Ecuador. \\ ${ }^{2}$ Universidad Técnica de Babahoyo Extensión Quevedo. Quevedo-Ecuador. \\ ${ }^{3}$ Universidad Técnica de Babahoyo Extensión Quevedo. Quevedo-Ecuador. \\ ${ }^{4}$ Universidad Técnica de Babahoyo Extensión Quevedo. Quevedo-Ecuador.
}

Contacto: mtubay@utb.edu.ec

\section{Resumen}

Las demandas academias como estudiar para un examen, revisar lecciones, ejecutar una asignación, elaborar un proyecto, preparar una exposición, entre otras; son invariables. Estas significan una carga académica y emocional para los estudiantes adolescentes, lo que significa que estos pueden cumplir o no con dicha labor a la vez que evalúan cómo la logran cumplir, sea a último momento, con ayuda, sin entender la tarea, etc. El ejercicio de evadir, postergar, rehuir o justificar retrasos hace referencia a la procrastinación, en la que algunos estudiantes secundarios suelen preguntarse: ¿será esto lo que quiero hacer durante mi vida?, ¿lograré realizar estas tareas sin el apoyo de los docentes?, ¿valgo para esto?, ¿tendré suficientes conocimientos para entrar a la universidad? La presente investigación trató de demostrar la influencia que tiene la procrastinación académica en la formación de la identidad en 
los adolescentes de la Unidad Educativa La Maná y considerar algunas concepciones concernientes a la personalidad y sus características. Además, se provee de algunos resultados de la misma, la cual puede proporcionar datos relevantes tanto para padres como para docentes sobre la situación actual de la posposición de tareas académicas.

También, aporta mayor información práctica sobre la dependencia entre la procrastinación académica y las personalidades de los estudiantes que participaron de la encuesta en función de su edad, manifestando si el aplazamiento académico restringe el tiempo dedicado al estudio, y si esta disminución se puede dar por el factor edad, por el factor curso académico, o por una combinación de ambas variables.

Palabras clave: Procrastinación Académica, Ansiedad, Personalidad, Identidad.

\begin{abstract}
The academies demand such as studying for an exam, reviewing lessons, executing an assignment, preparing a project, preparing an exhibition, among others; they are invariable. These represent an academic and emotional burden for adolescent students, which means that they may or may not fulfill this task while evaluating how they manage to accomplish it, be it at the last minute, with help, without understanding the task, etc. The exercise of avoiding, postponing, avoiding or justifying delays refers to procrastination, in which some high school students often ask themselves: is this what I want to do during my life? Will I be able to carry out these tasks without the support of teachers? Am I good for this? Will I have enough knowledge to enter university? The present research tried to demonstrate the influence that academic procrastination has on the formation of identity in adolescents from the La Maná Educational Unit and to consider some conceptions concerning personality and its characteristics. In addition, some results of the same are provided, which can provide relevant data for both parents and teachers on the current situation of the postponement of academic tasks. It also provides more practical information on the dependence between academic procrastination and the personalities of the students who participated in the survey based on their age, stating whether academic postponement restricts the time devoted to study, and if this decrease can be given by the age factor, by the academic year factor, or by a combination of both variables.
\end{abstract}


Keywords: Academic Procrastination, Anxiety, Personality, Identity

\section{Introducción}

Algunos de las situaciones primordiales que afrontan los estudiantes adolescentes son el conflicto para realizar actividades académicas dentro de un término de tiempo determinado, así como reconocer sus niveles de agitación y ansiedad durante un contexto de evaluación. La procrastinación es una predisposición muy divulgada entre la población occidental, mediante la cual se aplaza el inicio y/o finalización de trabajos que podrían efectuarse en un tiempo señalado y que se acompaña de sentimientos de incomodidad, sin embargo, hay que considerar que, no toda conducta de postergación o aplazamiento implica procrastinación.

Para Pardo, Perilla, \& Salinas (2014) la procrastinación es un comportamiento que se asocia con: cambios, incomodidades, exigencias ambientales y/o aversión al cumplimiento, bien sean en el ámbito académico, familiar, laboral, entre otros, como consecuencias causales o probabilísticas, es decir, es la evitación innecesaria del trabajo, lo que implica una disminución de esfuerzo y responsabilidad, constituyéndose en un hábito peligroso.

Todas las definiciones incorporan dos elementos: marco temporal y malestar subjetivo. El aplazamiento de la conducta genera un sentimiento de malestar en la persona porque no inicia y/o finaliza la tarea en el tiempo establecido. A nivel cognitivo, la persona genera todo tipo de sesgos para preservar su nivel de autoestima y no sentirse valorada por los demás; a nivel conductual, es probable que se implique en tareas que nada tienen que ver con el cumplimento de la tarea objetivo, constituyendo toda una suerte de conductas de auto-sabotaje distractoras.

Mamani (2017) enfatiza que la procrastinación implica que la persona ha planificado realizar la tarea en cierto tiempo y que por diferentes motivos no ha llegado a concluirla por lo que se evidencia que sí hubo planificación de tareas más no la ejecución de estas. La diferencia entre una persona procrastinadora y una persona no procrastinadora radica en que la segunda planifica atrasar la tarea; si la persona ha planificado atrasar la tarea por diferentes motivos, pero contempla realizarlo, es una persona no procrastinadora. 
Una cantidad considerable de estudiantes afrontan a diario una subyugada capacidad para resistirse a los incentivos sociales, las redes sociales, actividades agradables y satisfacciones inmediatas cuando los beneficios del desarrollo académico son separados y poco satisfactorios, lo que se ve manifestado en problemas para planear, establecer prioridades, organizarse, procurar atención a los complementos y recordar lo que tienen que hacer en un período explícito.

Tesouro, Palomanes, Bonachera, \& Martínez (2013) refieren que, se debe dar valor a la identidad de un grupo de adolescentes para poder hacer una categorización y ver las posibles diferencias en los estatus identitarios entre los alumnos situados en una adolescencia inicial y los situados en una adolescencia tardía. También se debe considerar las posibles diferencias asociadas al género.

En esta sociedad que está en permanente cambio -y que en las últimas décadas se ha modificado paradigmáticamente el estilo de vida de sus integrantes- se encuentran los adolescentes., ya que la adolescencia en sí misma implica vulnerabilidad y es allí donde más se hacen sentir las nuevas condiciones (Vázquez \& Fernández, 2016).

A nivel emocional, el sentimiento de infravaloración, ansiedad, y afectividad, genera una tendencia a la pasividad que no es energía o motiva para acometer la tarea. A nivel social, las personas que procrastinan son indecisas en sus relaciones interpersonales, pensando constantemente en la opinión que los demás pueden tener de uno/a misma, y cuestionando constantemente los modos tradicionales y establecidos de hacer las cosas. El tratamiento de la procrastinación implica realizar algo más que un curso de gestión de tiempo.

Vázquez \& Fernández (2016) destacan tambien que, durante la etapa de transición en el desarrollo humano, se produce una serie de cambios que implican un proceso de renuncia de la identidad infantil por la construcción de la identidad de adulto. El joven adolescente se va despojando de la capa protectora familiar de la que se compone esta identidad primaria y se asoma al mundo adulto en carne viva para construir - a prueba y error- ante la mirada de sus pares, su nueva identidad.

Por lo tanto, como ha quedado patente en diversas investigaciones, debe abordarse estas peculiaridades, desde la esfera cognitiva, afectiva y conductual, identificando el estilo de personalidad 
que procrastina, y ajustando el tratamiento a sus peculiaridades; las diferencias individuales, así pues, hacen que las recetas generales no sean eficaces ni eficientes.

Por último, Esteban, Nadal, Vila, \& Rostan (2013) señalan que, los espacios narrativos de la identidad: donde lo público deviene privado, la identidad personal y colectiva es el resultado de los relatos que contamos, a los otros y a nosotros mismos, sobre quiénes somos; integrando (dando sentido) al pasado y anticipando (proyectando) el porvenir. La relación con el medio está siempre mediada culturalmente, es decir, mediante los instrumentos psicológicos y culturales (el lenguaje, el más importante de ellos) se apropian de los espacios.

La posposición de actividades académicas o de cualquier índole, es inseparable a la vida actual, por lo que no se debería conceptuar a los sujetos, sus acciones, relaciones, identidades, separadas de su personalidad e identidad ya que la falta de conocimiento sobre este contexto, ha llevado a la creación de una serie de ficciones, que la relacionan a conductas antisociales, sedentarismo, etc.

\section{Materiales y métodos}

La investigación tuvo el nivel de asociación de variables porque permitió constituir predicciones a través de la intervención de las relaciones entre variables, midiendo su grado de relación y a partir de ello, determinar tendencias o modelos de comportamiento mayoritario. El presente trabajo sostiene la modalidad Prospectiva, ya que buscó la información necesaria para establecer la influencia de la procrastinación académica y la formación de la identidad en los adolescentes de la Unidad Educativa La Maná.

Además, se utilizó el método Hipotético-Deductivo, que determinó la lógica para dar conclusiones, que se establecieron en hechos admitidos como verdaderos, para llegar a conclusiones, donde sus aplicaciones sean de carácter general. El método dialectico permitió resaltar aquellos elementos de un fenómeno, procediéndolos a revisar ordenadamente cada uno de ellos por separado, permitiendo distinguir los elementos de un fenómeno y proceder a revisar ordenadamente cada uno de ellos.

Las técnicas utilizadas para reunir la información se ajustaron al ejemplo cuali-cuantitativo, ya que además de estudiar al ser humano en su cotidianidad, se establecerán resultados y conclusiones 
detalladas. La observación permitió legitimar claramente en el aula de clases si existen casos específicos de estudiantes con procrastinación académica, además de corroborar si esto incide negativamente en la formación de la identidad de los estudiantes, impidiéndoles que reconozcan y desarrollen competencias, habilidades y conocimientos.

Como instrumento se utilizó el formulario de encuestas, en los que se abordan datos de identificación de los pobladores, una guía de grupo focal que estará conformado por estudiantes, padres de familia y docentes, para determinar el nivel de conocimiento sobre el tema de estudio.

\section{Resultados}

El trabajo de investigación está delimitado en el cantón La Maná 4. Este cantón está ubicado a unos $150 \mathrm{~km}$ de Latacunga, capital de la provincia. Es una zona agrícola considerada la cuarta zona exportadora de banano. Limita al norte con la parroquia Alluriquín, cantón Santo Domingo; al Sur con el río Calope, accidente geográfico que la separa de la parroquia Moraspungo, cantón Pangua, al este con la parroquia El Tingo del cantón Pujilí y Sigchos y al oeste con el cantón Valencia y Quinsaloma de la provincia de Los Ríos.

La población es el grupo de individuos que habitan en una determinada zona donde se realiza la investigación. En la presente investigación se consideró una población de estudio determinada en la totalidad por estudiantes, padres de familia y docentes de la Unidad Educativa "La Maná". Se tomó en consideración una población de 60 estudiantes, 60 padres de familia y 10 docentes, la misma que facilitó el estudio y la investigación.

\begin{tabular}{|l|c|c|c|c|}
\hline INVOLUCRADOS & POBLACIÓN & $\%$ & MUESTRA & $\%$ \\
\hline Estudiantes & 60 & $47 \%$ & 60 & $47 \%$ \\
\hline Padres de familia & 60 & $47 \%$ & 60 & $47 \%$ \\
\hline Docentes & 10 & $6 \%$ & 10 & $6 \%$ \\
\hline Total & 130 & 100 & 130 & 100 \\
\hline
\end{tabular}


Tabla 1. Población de la comunidad educativa de la Unidad Educativa "La Maná".

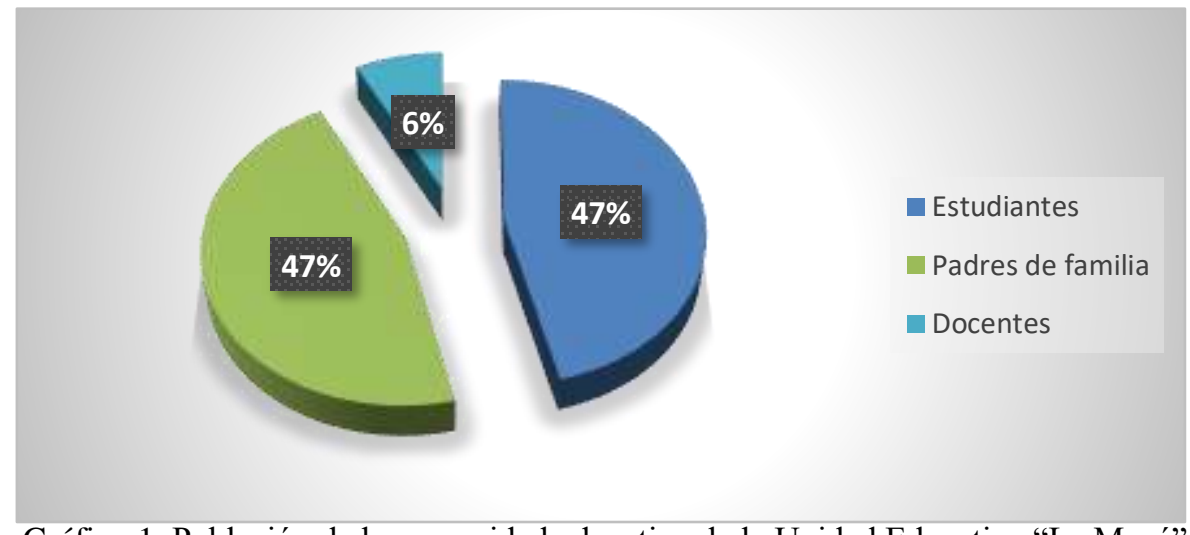

Gráfico 1. Población de la comunidad educativa de la Unidad Educativa "La Maná”.

\section{Encuesta dirigida a los estudiantes de la Unidad Educativa "La Maná"}

¿Considera que le preocupa demasiado la idea de obtener una calificación negativa?

\begin{tabular}{|l|c|c|}
\hline \multicolumn{1}{|c|}{ ALTERNATIVA } & FRECUENCIA & PORCENTAJE \\
\hline Nunca & 18 & $30 \%$ \\
\hline Algunas veces & 19 & $32 \%$ \\
\hline Muy frecuentemente & 23 & $38 \%$ \\
\hline TOTAL & 60 & $100 \%$ \\
\hline
\end{tabular}

Tabla 2. Preocupación por calificaciones negativas en sus asignaturas

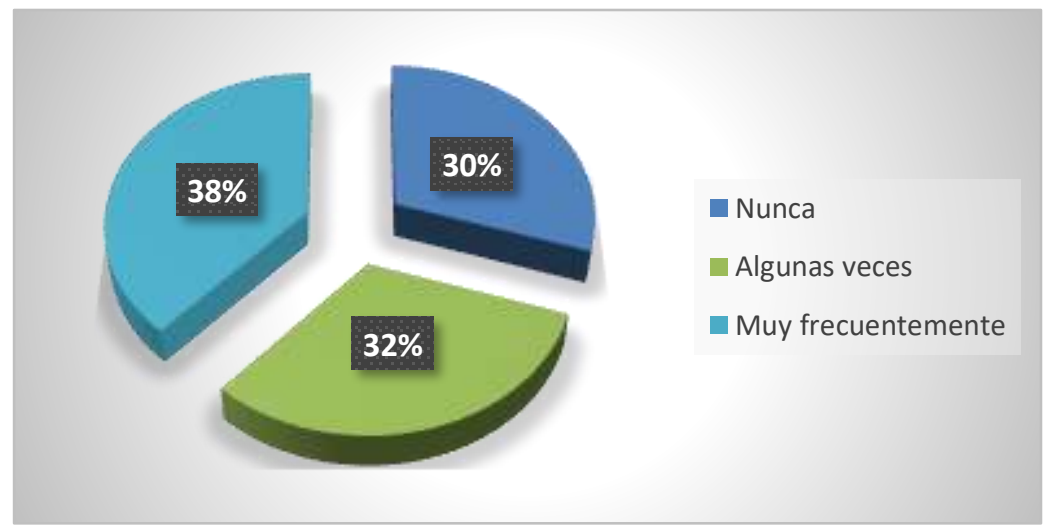

Gráfico 2. Preocupación por calificaciones negativas en sus asignaturas

De los estudiantes encuestados, el $30 \%$ afirmó que nunca se preocupa demasiado pensando en que se podría sacar una mala calificación, el 32\% destacó que algunas veces y el restante 38\% muy frecuente 
está preocupado sobremanera por su rendimiento académico. Es importante que los padres de familia, junto con los docentes, fomenten en el estudiante la necesidad de cumplir con sus actividades académicas, pero sin que estas, limiten la interacción en los demás campos sociales de los alumnos.

Cerda, Salazar, Guzmán, \& Narváez (2018) señala que, desde esta perspectiva, la escuela no es sólo un escenario de formación y entrega de conocimientos, sino que se legitima como un espacio para promover actitudes y valores encaminados a desarrollar una convivencia pacífica entre los seres humanos, por lo que la convivencia implica una trama compleja de diversas relaciones entre los actores que participan del proceso educativo de un establecimiento, constituyendo dinámicas de relación y poder que influyen en el clima emocional, estilos comunicacionales y vinculares, que son propios del lugar donde se reproducen.

¿Cree que pierde tiempo en trivialidades antes de tomar la decisión de estudiar?

\begin{tabular}{|l|c|c|}
\hline ALTERNATIVA & FRECUENCIA & PORCENTAJE \\
\hline Nunca & 23 & $38 \%$ \\
\hline Algunas veces & 21 & $35 \%$ \\
\hline Muy frecuentemente & 16 & $27 \%$ \\
\hline TOTAL & 60 & $100 \%$ \\
\hline
\end{tabular}

Tabla 3. Pérdida de tiempo en trivialidades antes de decidir estudiar

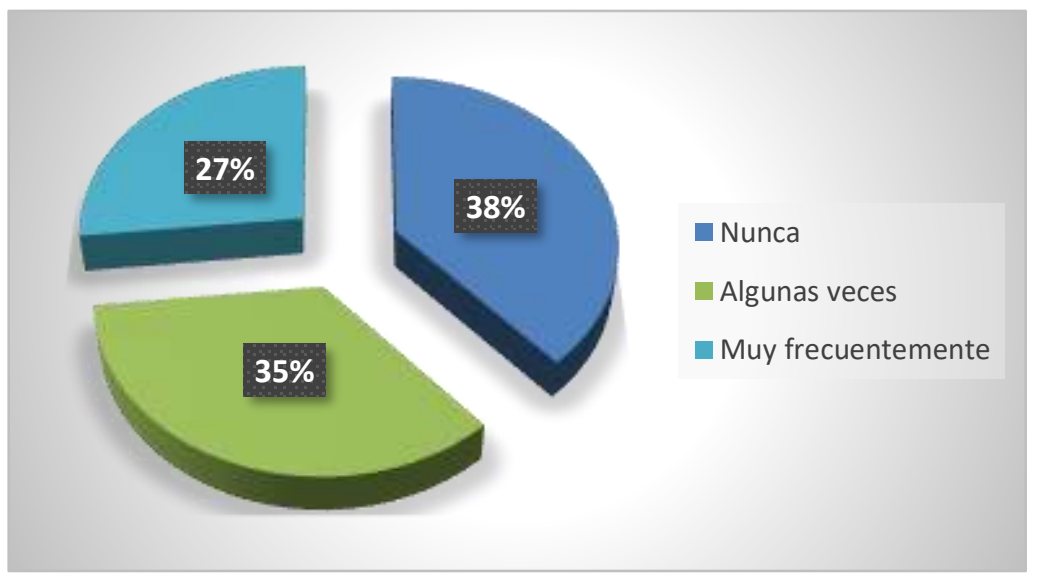

Gráfico 3. Pérdida de tiempo en trivialidades antes de decidir estudiar

El 38\% de los encuestados cree que pierde bastante tiempo en detalles sin importancia antes de tomar la decisión final, 35\% contestaron que algunas veces y el restante $27 \%$ afirmaron que muy 
frecuentemente gastan mucho tiempo analizando ciertas situaciones. Rodríguez \& Clariana (2017) afirman que se puede pensar que las conductas dilatorias están relacionadas con una falta de propósitos o motivación, pero la mayoría de investigadores creen que la postergación no es intencional. Es decir, los estudiantes que se motivan por el logro de un objetivo y no por el placer que produce el estudio en sí mismo, pero que perciben la decisión de estudiar como propia, presentan menos conductas dilatorias en sus estudios.

Pero esta incapacidad de terminar los proyectos no se debe a una falta de motivación. Los procrastinadores académicos, según las autoras citadas, son alumnos motivados que no tienen intención de estudiar menos o más tarde que los alumnos no procrastinadores, por lo que el motivo de su procrastinación es la falta de capacidad para alejar tentaciones y distracciones durante su estudio.

\section{Encuesta dirigida a los padres de familia de la Unidad Educativa "La Maná"}

\section{¿Considera que su hijo pospone actividades académicas que se había propuesto realizar con anterioridad?}

\begin{tabular}{|l|c|c|}
\hline ALTERNATIVA & FRECUENCIA & PORCENTAJE \\
\hline Nunca & 22 & $37 \%$ \\
\hline Algunas veces & 17 & $28 \%$ \\
\hline Muy & 21 & $35 \%$ \\
\hline \multicolumn{1}{|c|}{ TOTAL } & 60 & $100 \%$ \\
\hline
\end{tabular}

Tabla 4. Pospone actividades académicas que se había propuesto realizar con anterioridad.

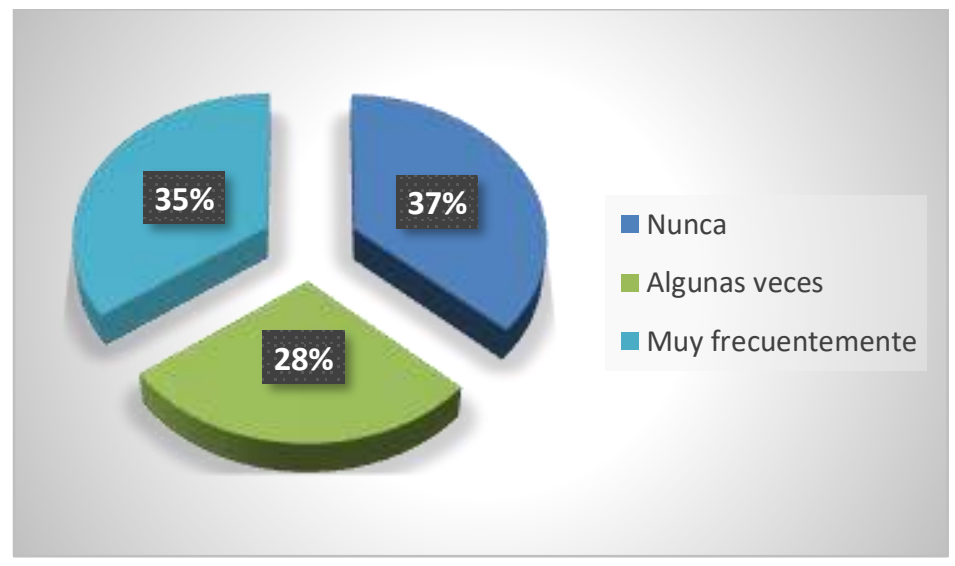

Gráfico 4. Pospone actividades académicas que se había propuesto realizar con anterioridad. 
De los padres de familia encuestados, el $37 \%$ considera que su hijo frecuentemente se da cuenta que está haciendo tareas que se había propuesto hacer con anterioridad, el 28\% en algunas ocasiones y el restante $35 \%$ señalan que muy frecuentemente se percata de que su hijo realiza tareas atrasadas. Matalinares, y otros (2017) confirman que, la gestión del tiempo y método general del trabajo sería un indicador importante para medir la conducta procrastinadora, debido a que parece lógico que aquellas personas con un mayor nivel de ansiedad ante la vida sean más proclives a procrastinar, identificando que la presencia de estrés es relevante como indicador de procrastinación.

Ahora bien, según los autores, con la aparición de numerosos instrumentos y soportes que han revolucionado la forma de manejar, almacenar y transmitir la información, se han producido importantes cambios en la forma de trabajar, en la forma de relacionarnos con otras personas, en la vida cotidiana y en las actividades de ocio.

\section{¿Considera que su hijo no presta atención cuando le habla claramente?}

\begin{tabular}{|l|c|c|}
\hline \multicolumn{1}{|c|}{ ALTERNATIVA } & FRECUENCIA & PORCENTAJE \\
\hline Nunca & 18 & $30 \%$ \\
\hline Algunas veces & 26 & $43 \%$ \\
\hline Muy frecuentemente & 16 & $27 \%$ \\
\hline TOTAL & 60 & $100 \%$ \\
\hline
\end{tabular}

Tabla 5. No presta atención cuando le habla claramente.

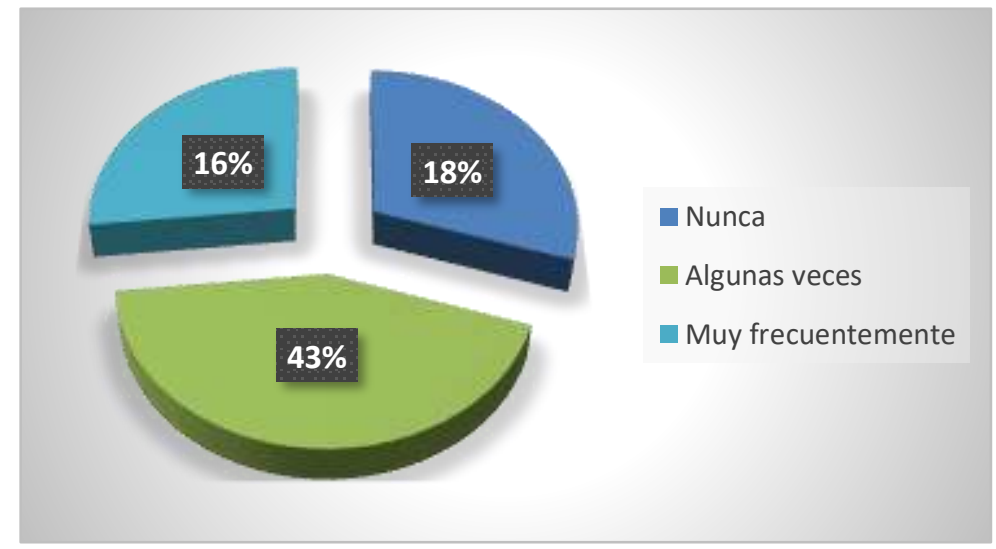

Gráfico 5. No presta atención cuando le habla claramente.

De los encuestados el $30 \%$ afirmó que sus representados no prestan atención cuando se le habla directamente, el $43 \%$ consideró que algunas veces y el 27\% destacó que muy frecuentemente ocurre 
esto con su hijo. Para que un estudiante adolescente logre tener éxito en su desempeño académico, debe contar con el apoyo de sus progenitores, que no solo deben limitarse al control y revisión de tareas, sino, además, en el apoyo y preocupación constante hacia todas las actividades que debe realizar su representado.

Estos pensamientos negativos de anticipación de un resultado de fracaso interferirían con la ejecución de la tarea, pues la persona desviaría su atención a este posible resultado y no podría concentrarse en lo aprendido para el examen.

Es importante indicar que las razones por las que las personas perciben el peligro en estas situaciones pueden ser diversas y muchas se basan en interpretaciones erróneas sobre ellas mismas, por lo que esos pensamientos negativos podrían no siempre tener un fundamento real (Vallejos, 2015).

\section{Encuesta dirigida a los docentes de la Unidad Educativa "La Maná"}

\section{¿A sus estudiantes se le dificulta seguir instrucciones de principio a fin y no termina el trabajo establecido?}

\begin{tabular}{|l|c|c|}
\hline ALTERNATIVA & FRECUENCIA & PORCENTAJE \\
\hline Nunca & 2 & $20 \%$ \\
\hline Algunas veces & 4 & $40 \%$ \\
\hline Muy & 4 & $40 \%$ \\
\hline TOTAL & 10 & $100 \%$ \\
\hline
\end{tabular}

Tabla 6. Seguir instrucciones de principio a fin y no termina el trabajo establecido.

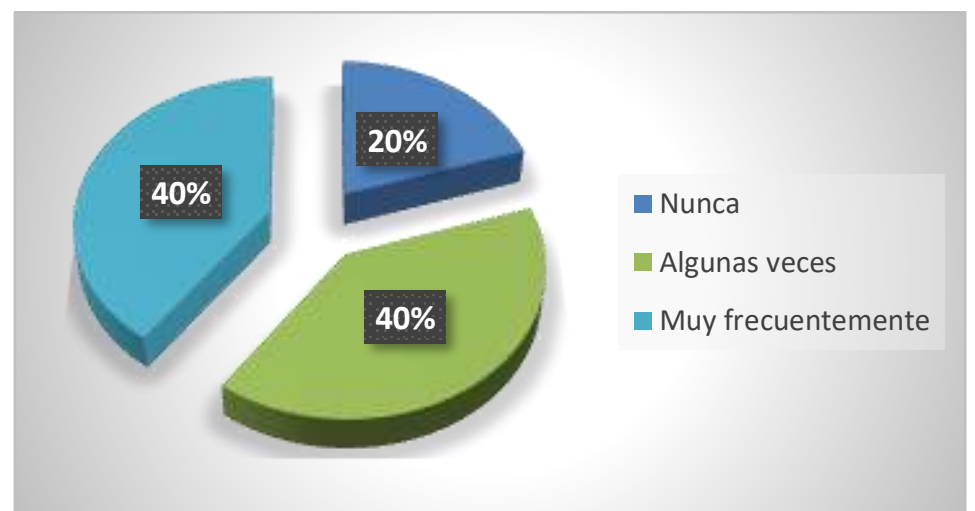

Gráfico 6. Seguir instrucciones de principio a fin y no termina el trabajo establecido. 
De los docentes encuestados, el 20\% afirmó que sus estudiantes se le dificulta seguir instrucciones de principio a fin y no termina el trabajo establecido, el $40 \%$ algunas veces y el restante $40 \%$ muy frecuentemente observa que algunos de sus estudiantes tienen este tipo de dificultades. El docente siempre debe apoyarse en los representantes de sus estudiantes, para obtener la cooperación necesaria que lleve al éxito académico de sus estudiantes.

Según Gaete (2015) el conocimiento y consideración del grado de desarrollo psicosocial del joven no solo son importantes para supervisar que el crecimiento y desarrollo se estén dando de manera normal, sino que tienen una incidencia determinante en los diversos aspectos de su atención; de este dependerán, entre otras muchas cosas, el tipo de lenguaje que se tendrá que utilizar, la sintomatología que podrá relatar, los niveles de confidencialidad a garantizar al joven, la conciencia de problema y grado de motivación al cambio que podrá esperarse, el nivel de competencia que se le reconocerá para la toma de decisiones respecto de su salud, en qué medida podrá responsabilizársele de las indicaciones y cuánto se deberá involucrar a los padres en el manejo.

Según su percepción, ¿sus estudiantes presentan problemas para organizar las tareas y actividades que debe llevar a cabo?

\begin{tabular}{|l|c|c|}
\hline \multicolumn{1}{|c|}{ ALTERNATIVA } & FRECUENCIA & PORCENTAJE \\
\hline Nunca & 3 & $30 \%$ \\
\hline Algunas veces & 5 & $50 \%$ \\
\hline Muy frecuentemente & 2 & $20 \%$ \\
\hline TOTAL & 10 & $100 \%$ \\
\hline
\end{tabular}

Tabla 7. Presentan problemas para organizar las tareas y actividades.

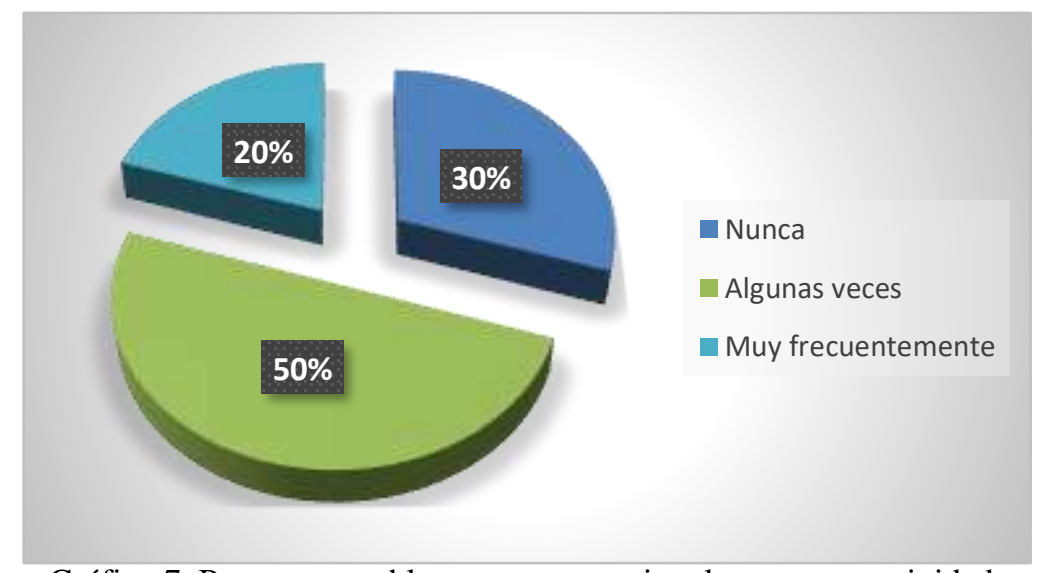

Gráfico 7. Presentan problemas para organizar las tareas y actividades. 
El 30\% de encuestados aseveraron que nunca ocurre que sus estudiantes presentan problemas para organizar las tareas y actividades que debe llevar a cabo, el 50\% afirmó que algunas veces y el 20\% contestó que muy frecuentemente. Vázquez \& Fernández (2016) afirmaron que, la identificación, un imprescindible en la construcción de identidad La identidad es una construcción subjetiva en la cual el Otro es condición de posibilidad. Su configuración se va diseñando por medio de un proceso de identificaciones que el sujeto realiza desde los primeros años de vida y se delinea en su historia en relación con los otros.

Los docentes deben trabajar junto a los padres de familia y estar en constante comunicación para que se puedan resolver los imponderables que puedan surgir en los procesos académicos que se desarrollan en el aula de clases.

La identidad es por un lado un sí mismo único y también puede ser varios nosotros acorde con los roles que se asuman socialmente; la que distingue un grupo o comunidad de otros y una individualidad de otras, no es estática, va cambiando y supone alteridad, al darse por la experiencia con los otros diferentes- en el tiempo (Vázquez \& Fernández, 2016).

\section{Discusión}

La vida secundaria exige recursos personales como gestión del tiempo y habilidades sociales, y recursos cognitivos como resolución de problemas y pensamiento crítico - reflexivo, por lo que estas demandas se pueden exteriorizar en los alumnos como un estado amenazador o estado de alerta, que por lo general se conoce como ansiedad.

Esta es una característica recurrente en la mayoría de los estudiantes adolescentes que se enfrentan a nuevas tareas que tienen que ver con su vida, ya que no solo tienen responsabilidades académicas, sino que atraviesan una serie de cambios de carácter económico, personal, emocional, etc., a los cuales se les suman los esfuerzos por tratar de llevar y mantener un promedio ventajoso para su futuro laboral

Para los estudiantes, el hecho de posponer las tareas supone un problema y a su vez, incrementa la posibilidad de manifestación de reacciones fisiológicas relacionadas al estrés y a la ansiedad, por lo 
que se evidencia que a mayor postergación de actividades académicas que realizan los estudiantes presentan mayores niveles de ansiedad (Gil \& Botello, 2018).

Según las autoras citadas, existe una asociación significativa con el hecho de posponer las tareas, lo cual supone un problema a nivel académico y a su vez, incrementa la posibilidad de manifestación de reacciones fisiológicas relacionadas a la ansiedad, al estar expuestos a las exigencias académicas; tales como, el desafío de combinar sus estudios con situaciones familiares, que implican constantes cambios del ciclo de sueño-vigilia, la exigencia de los docentes y la relación con su entorno social.

(Colás \& Villaciervos, 2013) argumentan que, las personas se convierten en hombres y mujeres en función del aprendizaje de representaciones culturales de género que rigen, no sólo, su constitución genérica, sino también, el carácter de las relaciones que, unos y otras, mantienen en diferentes esferas sociales (en ámbitos como la familia, la escuela, el grupo desiguales, etc.). Así, el género, como sistema cultural, provee de referentes culturales que son reconocidos y asumidos por las personas.

En la sociedad occidental las personas habitualmente se plantean metas a corto, medio y largo plazo relacionadas con uno mismo/a, la educación, el trabajo, la salud, etc. lo cual implica trazar un plan para conseguirlas, ya sea un plan explícito o implícito, y una estimación del tiempo necesario para realizarlo.

Díaz (2019) afirma que, la procrastinación consiste en la tendencia generalizada a aplazar el inicio y/o finalización de tareas planificadas para ser realizadas en un tiempo determinado. Tal tendencia a la postergación suele acompañarse de malestar subjetivo y no sólo es una cuestión de baja responsabilidad y gestión del tiempo, sino que supone un verdadero problema de auto-regulación a nivel cognitivo, afectivo y conductual.

Si las actividades que se planean realizar se llevan a cabo en tiempo, muy probablemente las consecuencias positivas de tales actividades sean evidentes; por el contrario, si las conductas planeadas y orientadas a futuro no se realizan en el tiempo estimado, la probabilidad de que aparezcan consecuencias negativas se incrementa (Díaz, 2019). 
Por otro lado, Zuazagoitia, Echeazarra, Ros, \& Infante (2016) sostienen que, aplazar la realización de una determinada actividad en favor de otra es una práctica muy humana que se conoce como procrastinar. La procrastinación es una variable universal y atemporal propia comportamiento humano, tal y como reflejan diversos textos antiguos que hace miles de años hacían referencia a este concepto.

Según los autores en referencia, por lo general, las personas que "dejan para mañana lo que pueden hacer hoy" son vistas como personas perezosas, vagas o irresponsables, así valoradas incluso por otras personas con la misma tendencia. La falta de diligencia a la hora de realizar tareas planeadas en un tiempo estimado se asocia con cierto malestar subjetivo, y si tal tendencia se da en diferentes áreas vitales, entonces se trata de una persona que procrastina.

Varios autores han resaltado que los procrastinadores tienden a postergar las tareas ante la necesidad de disponer de tiempo para poder pensar, por lo que ser procrastinador estaba bien considerado, sin embargo, la procrastinación es un comportamiento que ha adquirido una connotación negativa en el marco de las sociedades que emergieron de la revolución industrial.

Actualmente, la costumbre de postergar asuntos pendientes no está bien vista, tal y como refleja el refranero popular con dichos como "no dejes para mañana lo que puedas hacer hoy". Se vive en una sociedad que tiene muy en cuenta las tasas de productividad en cualquier ámbito de la vida, por lo que aplazar los asuntos pendientes se traduce en elevados costos asociados a pérdidas de productividad. Como consecuencia de lo anterior, la procrastinación es entendida actualmente como un trastorno del comportamiento que tiene su raíz en la asociación de la acción a realizar con el cambio, el dolor o la incomodidad (estrés).

Este puede ser psicológico (en la forma de ansiedad o preocupación), físico (como el que se experimenta durante los actos que requieren trabajo duro o vigoroso), por lo que a este comportamiento consistente en retrasar la acción se le atribuye una falta de regulación conductual con consecuencias desagradables para el sujeto (Zuazagoitia, Echeazarra, Ros, \& Infante, 2016).

Por lo tanto, este retraso intencionado, según los autores en referencia, pretende propiciar una sensación de motivación y optimización de recursos que se suelen encontrar en la situación final de la 
dilación, por lo que hay que tener en cuenta que la procrastinación es una conducta que se manifiesta en diferentes ámbitos de la vida de las personas, siendo estudiada en gran profundidad especialmente en dos ámbitos: el académico y el laboral.

\section{Conclusiones}

El aplazar las tareas académicas o las labores diarias interviene en los hábitos y atención de las personas, además de la motivación, razón por la que los procrastinadores tienden a demorar las tareas que perciben como aversivas, de escaso valor, que se distinguen como difíciles de realizar o ante las cuales no se identifican como capaces de lograr o que se relacionan con menores o dilatadas recompensas

La procrastinación académica incluye la disposición de evitar hacer algo a pesar de tener el conocimiento que a largo plazo empeora la situación que no se atiende, por lo que no es lo mismo que atrasar intencionalmente alguna actividad, ya que no es un asunto de manejo de tiempo, sino una incapacidad de controlar las emociones e impulsos.

En el ambiente académico, la procrastinación llega a ser la suspensión de tareas del contexto escolar, encontrándose que esta podría estar relacionada con la presencia de comportamientos de aplazamiento en otras áreas evidenciando en el estudiante una baja autoestima, déficit en autoconfianza, déficit de autocontrol, depresión y en algunos casos perfeccionismo, ímpetu disfuncional y ansiedad.

La procrastinación académica es un tema de utilidad tanto para psicólogos, educadores, como para los propios estudiantes, por lo que una propuesta enfocada en una intervención psicológica será de ayuda para hallar soluciones firmes para el control del aplazamiento injustificado en la elaboración de las tareas en el ambiente educativo.

\section{Bibliografía}

Cerda, G., Salazar, Y., Guzmán, C., \& Narváez, G. (2018). Impacto de la convivencia escolar sobre el rendimiento académico, desde la percepción de estudiantes con desarrollo típico y necesidades educativas especiales. Propósitos y Representaciones, 6(1), 247-300. 
Colás, P., \& Villaciervos, P. (2013). La interiorización de los estereotipos de género en jóvenes y adolescentes. Revista de Investigación Educativa, 35-58.

Díaz, J. (2019). Procrastinación: Una Revisión de su Medida y sus Correlatos. Revista Iberoamericana de Diagnóstico y Evaluación-e Avaliação Psicológica. RIDEP, 2(51), 43-60.

Esteban, M., Nadal, J., Vila, I., \& Rostan, C. (2013). Aspectos ambientales implicados en la construcción de la identidad en una muestra de adolescentes de la Universidad Intercultural de Chiapas. Medio Ambiente y Comportamiento Humano, 91-117.

Gaete, V. (2015). Desarrollo psicosocial del adolescente. Revista Chilena de Pediatría, 86(6), 436443.

Gil, L., \& Botello, V. (2018). Procrastinación académica y ansiedad en estudiantes de Ciencias de la Salud de una Universidad de Lima Norte. CASUS, 3(2), 89-96.

Mamani, S. (2017). Relación entre la procrastinación académica y ansiedad-rasgo en estudiantes universitarios pertenecientes al primer año de estudios de una universidad privada de Lima Metropolitana. Facultad de Psicología Leopoldo Chiappo Galli. Lima-Perú: Universidad Peruana Cayetano Heredia.

Matalinares, M., Díaz, A., Rivas, L., Dioses, A., Arenas, C., Villalba, O., . . Yaringaño, J. (2017). Procrastinación y adicción a redes sociales en estudiantes universitarios de pre y post grado de Lima. Horizonte de la Ciencia, 7(13), 63-81.

Pardo, D., Perilla, L., \& Salinas, C. (2014). Relación entre procrastinación académica y ansiedadrasgo en estudiantes de psicología. Cuadernos Hispanoamericanos de Psicología, 14(1), 31-44.

Rodríguez, A., \& Clariana, M. (2017). Procrastinación en estudiantes universitarios: su relación con la edad y el curso académico. Revista Colombiana de Psicología, 26(1), 45-60.

Tesouro, M., Palomanes, M., Bonachera, F., \& Martínez, L. (2013). Estudio sobre el desarrollo de la identidad en la adolescencia. Tendencias Pedagógicas (21), 211-224. 
Vallejos, S. (2015). Procrastinación académica y ansiedad frente a las evaluaciones en estudiantes universitarios. Facultad de Letras y Ciencias Humanas. Lima-Perú: Pontificia Universidad Católica del Perú.

Vázquez, C., \& Fernández, J. (2016). Adolescencia y sociedad. La construcción de identidad en tiempos de inmediatez. PSOCIAL, 2(1), 38-55.

Zuazagoitia, A., Echeazarra, I., Ros, I., \& Infante, G. (2016). Procrastinación y actividad física: elaboración del cuestionario PAF. Ediciones : ACIPE- Asociación Científica de Psicología y Educación, 253-263. 